\title{
Maternal low-protein diet programmes low ovarian reserve in offspring
}

\author{
Amy L Winship, Sarah E Gazzard², Luise A Cullen-McEwen², John F Bertram² and Karla J Hutt ${ }^{1}$ \\ ${ }^{1}$ Department of Anatomy and Developmental Biology, Ovarian Biology Laboratory, Biomedicine Discovery Institute, \\ Monash University, Melbourne, Australia and ${ }^{2}$ Department of Anatomy and Developmental Biology, Kidney \\ Developmental Programming and Disease Laboratory, Biomedicine Discovery Institute, Monash University, \\ Melbourne, Australia
}

Correspondence should be addressed to KJ Hutt; Email: karla.hutt@monash.edu

\begin{abstract}
The ovarian reserve of primordial follicle oocytes is formed during in utero development and represents the entire supply of oocytes available to sustain female fertility. Maternal undernutrition during pregnancy and lactation diminishes offspring ovarian reserve in rats. In mice, maternal oocyte maturation is also susceptible to undernutrition, causing impaired offspring cardiovascular function. We aimed to determine whether programming of the ovarian reserve is impacted in offspring when maternal undernutrition extends from preconception oocyte development through to weaning. C57BL6/J female mice were fed normal protein (20\%) or low-protein $(8 \%)$ diet during preconception, pregnancy and lactation periods. Maternal ovaries were harvested at weaning and offspring ovaries were collected at postnatal day (PN)21 and 24 weeks of age. Total follicle estimates were obtained by histologically sampling one ovary per animal ( $n=5$ /group). There was no impact of diet on maternal follicle numbers. However, in offspring, maternal protein restriction significantly depleted primordial follicles by $37 \%$ at PN21 and $51 \%$ at 24 weeks $(P<0.05)$. There were no effects of diet on other follicle classes. Histological analysis showed no differences in the proportion of proliferative follicles (pH3 positive), but increased atresia (cleaved caspase-3-positive, or TUNEL-positive) was detected in ovaries of protein-restricted offspring at both ages $(P<0.05)$. Our data show that maternal diet during the preconception period, in utero development and early life has significant impacts on follicle endowment and markers of follicle health later in life. This highlights the need for further investigation into the importance of maternal preconception diet for offspring reproductive development and health.

Reproduction (2018) 156 299-311
\end{abstract}

\section{Introduction}

The number and quality of oocytes stored within the ovary define the length of the female fertile lifespan (Wallace \& Kelsey 2010). Primordial follicle oocytes give rise to all mature oocytes and can remain quiescent in the ovary for months in mice, or decades in women, before being activated to undergo folliculogenesis and ovulation, or alternatively, atresia (McGee \& Hsueh 2000). A limited number of primordial follicles at a time are periodically activated to undergo folliculogenesis - a process characterised by dramatic growth of the oocyte and resumption of meiosis, as well as the extensive proliferation of the granulosa cells and ovulation of the dominant follicle(s). Folliculogenesis continues until the supply of primordial follicles is exhausted and menopause ensues.

Oocytes are formed during in utero development and then assembled into primordial follicles before birth, or shortly after, depending on the species (reviewed Findlay et al. 2015). Consequently, exposure to a suboptimal environment during foetal and early postnatal development, such as maternal undernutrition, has been shown to impair offspring ovarian reserve programming in animal models (Bernal et al. 2010, da Silva Faria et al. 2010a,b, Guzman et al. 2014). Some evidence suggests that exposure to malnutrition during maternal oocyte maturation prior to pregnancy can also impact offspring phenotypes. Maternal low-protein diet fed exclusively to female mice for one ovulatory cycle prior to mating impaired offspring cardiovascular function and led to behavioural abnormalities (Watkins et al. 2008), although the impacts of this on the ovary have not been examined.

In support though, unique evidence from a human cohort, namely the 'Dutch hunger winter families study' reported that maternal undernutrition prior to and during pregnancy resulted in early-onset menopause in their female children (Yarde et al. 2013). Whilst primordial follicle numbers cannot be determined in human populations in situ, these observations suggest that these females may have been born with an abnormally low pool of primordial follicles. 
Understanding the greatest period(s) of sensitivity of ovarian reserve formation to maternal malnourishment, however, has been complicated by methodological differences in animal studies, involving exposure to different diets, throughout different gestational and postnatal time points. Calorie restriction $(50 \%)$ in Wistar rats during pregnancy and lactation, but not either period alone, resulted in a significant $50 \%$ lower primordial follicle density in 150-day-old offspring (Bernal et al. 2010). Conversely, in a separate study, protein restriction during lactation only in Wistar rats resulted in a significant $60 \%$ lower primordial follicle density at puberty in 40-day-old offspring (da Silva Faria et al. 2010a), whilst only a $21 \%$ lower density was reported by the same authors in 90-day adult offspring (da Silva Faria et al. 2010b).

The findings from these studies highlight further inconsistencies in the approaches used to quantify ovarian follicles, which has hampered the comparison of findings between numerous ovarian programming studies and also any comparisons between species. Importantly, follicle density (number per volume), which is often reported as a surrogate measure for estimated total follicle number in the ovary, does not account for the unequal distribution of follicles throughout the ovary, or changes in ovarian volume, and often only samples a small fraction of the ovary.

To determine whether the sensitivity of offspring ovarian reserve programming extends to maternal oocyte maturation, we examined the combined effect of maternal protein restriction during preconception, pregnancy and lactation periods on offspring ovarian follicle endowment in vivo. Female C57BL6/J mice were fed normal or low-protein diet for 21 days prior to natural mating (the period of time required for development of a primordial follicle oocyte through maturation in mice) and were maintained on the diets throughout pregnancy and lactation. The number of primordial follicles and all other follicle classes were estimated in ovaries from control and protein-restricted mothers and offspring at PN21 and 24 weeks. Histological markers of ovarian follicle atresia, proliferation, DNA damage and mitochondria were also assessed.

\section{Materials and methods \\ Animals and tissue collection}

C57BL/6 mice were housed in a temperature-controlled high barrier facility (Monash University Animal Research Laboratories), with free access to food and water, under a 12-h light-darkness cycle. All animal procedures and experiments were performed in accordance with the NHMRC Australian Code of Practice for the Care and Use of Animals and approved by the Monash Animal Research Platform Animal Ethics Committee. Virgin female mice (10 weeks) were weight matched prior to being randomly allocated to standard AIN93G rodent diet as the control diet (20\% protein, Specialty Feeds,
Glen Forrest, Australia) (Table 1) or a low-protein modification of the AIN93G rodent diet (SF01-026; 8\% protein, Specialty Feeds, Glen Forrest) (Table 1) for 3 weeks (the period of time required for development of a primordial follicle oocyte through maturation in mice) prior to pairing with a male stud ( $n=11-12$ /group). At birth, the number of live pups and time to litter since pairing with a stud were recorded. Dams remained on the diet throughout pregnancy and lactation until weaning of offspring at postnatal day (PN)21. Only litters with a minimum of four pups and a maximum of nine pups were included. At PN21 (juvenile ovary), one female pup per litter and mothers were weighed and anesthetised by isoflurane (Bayer) inhalation and then cleared of blood by saline infusion before being perfusion fixed with $10 \%$ (vol/vol) neutral buffered formalin (Amber Scientific, Midvale, Australia) at a rate of $5 \mathrm{~mL} / \mathrm{min}$. Ovaries were harvested and fixed in $10 \%$ ( $\mathrm{vol} / \mathrm{vol}$ ) neutral buffered formalin solution for $24 \mathrm{~h}$ and paraffin embedded. One ovary from each female was used to obtain follicle counts and the contralateral ovary was used for TUNEL, immunohistochemical and immunofluorescence analysis. All remaining female offspring in the litter were fed normal chow diet from PN21 until 24 weeks of age (reproductively aged; still fertile) before being weighed and ovaries harvested as above.

\section{Follicle counts}

To estimate ovarian follicle numbers, paraffin-embedded ovaries were exhaustively sectioned at $5 \mu \mathrm{m}$ and stained with periodic acid-Schiff and haematoxylin ( $n=5$ /group) (SigmaAldrich). Whole tissue section images were captured on the DotSlide system at $\times 20$ objective using an XC10 camera (Olympus). The total number of primordial, transitional, primary follicles was quantified in every ninth section of each ovary and the total number of secondary and antral follicles were counted in every 36th section using a similar strategy as previously described (Tilly 2003, Hutt et al. 2006). Follicles were counted if the oocyte nucleus was present. Total follicle numbers were obtained by multiplying the raw counts of oocytes sampled (Q-) by nine to correct for the sections not counted. The number of corpora lutea was determined by direct counting of every 36th section encompassing the entire ovary. Adjacent sections were evaluated to ensure each corpora lutea was only counted once.

\section{Ovarian volume}

Estimation of total ovarian volume ( $n=5 /$ group) was performed according to the Cavalieri principle, at $\times 10$ magnification using a stereology system. Briefly, a test system of grid points was overlaid on the image of the tissue using Stereolnvestigator software (version 11.06.02, MBF Bioscience 2015) and repeated for every 18th section of the whole ovary as described previously (Karbalay-Doust \& Noorafshan 2012). Volume was calculated using the following equation: $V=\Sigma P \times a(p) \times t \times 1 / f$ where, $V$ is the volume of the ovary, $\Sigma P$ is the total number of grid points counted on complete and incomplete sections, $a(p)$ is the area associated with each grid point, $t$ is the section thickness and $1 / f$ is the reciprocal of the section sampling fraction. 
Table 1 Calculated nutritional parameters for control and low-protein diets (Specialty Feeds, Glen Forrest, Australia).

\begin{tabular}{|c|c|c|}
\hline Calculated nutritional parameter & Control diet & Low-protein diet \\
\hline Protein \% & 19.4 & 8.4 \\
\hline Total fat \% & 7 & 7 \\
\hline Crude fibre \% & 4.7 & 4.7 \\
\hline AD fibre $\%$ & 4.7 & 4.7 \\
\hline Digestible energy $(\mathrm{MJ} / \mathrm{kg})$ & 16.1 & 16.2 \\
\hline$\%$ Total calculated digestible energy from lipids & 16 & 16 \\
\hline$\%$ Total calculated digestible energy from protein $\%$ & 21 & 9 \\
\hline Casein (acid) $(\mathrm{g} / \mathrm{kg})$ & 200 & 87 \\
\hline Sucrose $(g / k g)$ & 100 & 200 \\
\hline Canola oil (g/kg) & 70 & 70 \\
\hline Cellulose $(g / k g)$ & 50 & 50 \\
\hline Wheat starch $(\mathrm{g} / \mathrm{kg})$ & 404 & 417 \\
\hline Dextrinised starch (g/kg) & 132 & 132 \\
\hline DL methionine $(\mathrm{g} / \mathrm{kg})$ & 3 & 3 \\
\hline Calcium carbonate $(\mathrm{g} / \mathrm{kg})$ & 13.1 & 13.1 \\
\hline Sodium chloride $(\mathrm{g} / \mathrm{kg})$ & 2.6 & 2.6 \\
\hline AIN93 trace minerals (g/kg) & 1.5 & 1.4 \\
\hline Potassium citrate $(\mathrm{g} / \mathrm{kg})$ & 2.5 & 2.5 \\
\hline Potassium dihydrogen phosphate (g/kg) & 6.9 & 6.9 \\
\hline Potassium sulphate $(\mathrm{g} / \mathrm{kg})$ & 1.6 & 1.6 \\
\hline Choline chloride $(75 \%)(\mathrm{g} / \mathrm{kg})$ & 2.5 & 2.5 \\
\hline AIN93 vitamins $(\mathrm{g} / \mathrm{kg})$ & 10 & 10 \\
\hline Valine $\%$ & 1.26 & 0.50 \\
\hline Leucine \% & 1.80 & 0.80 \\
\hline Isoleucine \% & 0.87 & 0.40 \\
\hline Threonine \% & 0.79 & 0.30 \\
\hline Methionine \% & 0.84 & 0.50 \\
\hline Cysteine \% & 0.05 & 0.03 \\
\hline Lysine \% & 1.49 & 0.60 \\
\hline Phenylalanine \% & 0.99 & 0.40 \\
\hline Tyrosine \% & 1.04 & 0.50 \\
\hline Histidine \% & 0.27 & 0.26 \\
\hline Tryptophan \% & 0.60 & 0.10 \\
\hline Calcium \% & 0.47 & 0.46 \\
\hline Phosphorous \% & 0.35 & 0.20 \\
\hline Magnesium \% & 0.08 & 0.10 \\
\hline Sodium \% & 0.15 & 0.14 \\
\hline Chloride \% & 0.16 & 0.16 \\
\hline Potassium \% & 0.40 & 0.40 \\
\hline Sulphur \% & 0.23 & 0.16 \\
\hline Iron (mg/kg) & 68 & 73 \\
\hline Copper (mg/kg) & 7.0 & 6.8 \\
\hline lodine $(\mathrm{mg} / \mathrm{kg})$ & 0.2 & 0.2 \\
\hline Manganese (mg/kg) & 19 & 25 \\
\hline Cobalt & No data & No data \\
\hline Zinc $(m g / k g)$ & 46 & 47 \\
\hline Molybdenum (mg/kg) & 0.15 & 0.15 \\
\hline Selenium $(\mathrm{mg} / \mathrm{kg})$ & 0.3 & 0.3 \\
\hline Cadmium & No data & No data \\
\hline Chromium (mg/kg) & 1.0 & 1.0 \\
\hline Fluoride (mg/kg) & 1.0 & 1.0 \\
\hline Lithium (mg/kg) & 0.1 & 0.1 \\
\hline Boron $(\mathrm{mg} / \mathrm{kg})$ & 3.3 & 1.9 \\
\hline Nickel (mg/kg) & 0.5 & 0.5 \\
\hline Vanadium $(\mathrm{mg} / \mathrm{kg})$ & 0.1 & 0.1 \\
\hline Vitamin A (Retinol) (IU/kg) & 4000 & 4000 \\
\hline Vitamin D (Cholecalciferol) (IU/kg) & 1000 & 1000 \\
\hline Vitamin E (a Tocopherol acetate) (mg/kg) & 78 & 75 \\
\hline Vitamin K (Menadione) $(\mathrm{mg} / \mathrm{kg})$ & 1 & 1 \\
\hline Vitamin C (Ascorbic acid) & None added & None added \\
\hline Vitamin B1 (Thiamine) (mg/kg) & 6.1 & 6.1 \\
\hline
\end{tabular}


Table 1 Continued.

\begin{tabular}{|c|c|c|}
\hline Calculated nutritional parameter & Control diet & Low-protein diet \\
\hline Vitamin B2 (Riboflavin) (mg/kg) & 6.3 & 6.3 \\
\hline Niacin (Nicotinic acid) (mg/kg) & 30 & 30 \\
\hline Vitamin B6 (Pryridoxine) (mg/kg) & 7 & 7 \\
\hline Pantothenic acid (mg/kg) & 16.5 & 16.5 \\
\hline Biotin $(\mu \mathrm{g} / \mathrm{kg})$ & 200 & 200 \\
\hline Folic acid (mg/kg) & 2 & 2 \\
\hline Inositol & None added & None added \\
\hline Vitamin B12 (Cyancobalamin) ( $\mu \mathrm{g} / \mathrm{kg})$ & 103 & 101 \\
\hline Choline (mg/kg) & 1470 & 1640 \\
\hline Myristic acid 14:0 & Trace & Trace \\
\hline Palmitic acid 16:0 \% & 0.30 & 0.30 \\
\hline Stearic acid 18:0 \% & 0.14 & 0.14 \\
\hline Palmitoleic acid 16:1\% & 0.02 & Trace \\
\hline Oleic acid 18:1 $3 \%$ & 3.89 & 3.99 \\
\hline Gadoleic acid 20:1 \% & 0.07 & 0.07 \\
\hline Linoleic acid 18:2 n6 \% & 1.51 & 1.51 \\
\hline a Linolenic acid 18:3 n3 \% & 0.98 & 0.98 \\
\hline Arachadonic acid 20:4 n6 & No data & No data \\
\hline EPA $20: 5$ n3 & No data & No data \\
\hline DHA 22:6 n3 & No data & No data \\
\hline Total n3 \% & 0.98 & 0.98 \\
\hline Total n6 \% & 1.51 & 1.51 \\
\hline Total mono unsaturated fats \% & 3.98 & 3.98 \\
\hline Total polyunsaturated fats \% & 2.50 & 2.50 \\
\hline Total saturated fats $\%$ & 0.50 & 0.50 \\
\hline
\end{tabular}

\section{TUNEL assay}

Paraffin-embedded ovaries were serially sectioned $(5 \mu \mathrm{m})$ and mounted on glass slides. The Apop Tag Peroxidase In Situ Apoptosis Detection Kit (Millipore) was used to detect apoptosis according to the manufacturer's instructions. Sections were counterstained with haematoxylin. Images were captured on a Provis AX70 Widefield microscope (Olympus). Five sections per ovary per mouse ( $n=5$ /group) were analysed in PN21 and adult (24 week old) offspring. Follicles were classified as positive if the oocyte and/or $\geq 4$ granulosa cells were positive when the ooplasm was present, as previously described (Flaws et al. 1997). The number of positive follicles was expressed as a proportion (\%) of total follicles.

\section{Immunohistochemistry and immunofluorescence}

Five tissue sections $(5 \mu \mathrm{m})$ per ovary were deparaffinised in histolene (Grale HDS, Ringwood, Australia), then rehydrated in a series of graded ethanol (Univar, Scoresby, Australia) ( $n=5$ /group). Antigen retrieval was performed by microwaving the sections for $10 \mathrm{~min}$ in $0.01 \mathrm{M}$ sodium citrate $(\mathrm{pH}$ 6) (SigmaAldrich). Endogenous peroxidases were quenched for $30 \mathrm{~min}$ in $0.3 \% \mathrm{H}_{2} \mathrm{O}_{2}$ (Univar, Scoresby, Australia), and then slides were washed $3 \times 5 \mathrm{~min}$ in $0.1 \mathrm{M}$ Tris, $150 \mathrm{mM} \mathrm{NaCl}$ and $0.1 \%$ v/v Tween buffer (TNT). Non-specific binding of antibodies was blocked by incubating the sections for $1 \mathrm{~h}$ with $10 \%$ normal goat serum (Sigma-Aldrich) in TNT. Sections were incubated with primary antibody against Phospho-Histone H3 (Phospho S10) (1:500; Abcam \#ab5176) or cleaved caspase-3 (D175) (1:200; Cell Signaling Technology \#9661S) in 1\% bovine serum albumin (BSA) (Sigma-Aldrich) in TNT overnight at $4{ }^{\circ} \mathrm{C}$ and later with biotinylated goat antibody against rabbit IgG (1:500;
Vector Laboratories, Burlingame, CA, USA) in 1\% BSA/TNT for $1 \mathrm{~h}$ at room temperature. Sections were incubated for $30 \mathrm{~min}$ with avidin-biotin peroxidase complex (Vector Laboratories). Peroxidase activity was visualised using 3,3'-diaminobenzidine (Dako) and sections were counterstained with haematoxylin (Sigma-Aldrich). Tissues stained with the primary antibody omitted were used as negative controls. Whole ovarian section $(\times 10$ objective) and high power images $(\times 40$ objective) were captured on the Provis AX70 Widefield microscope (Olympus). For immunofluorescence staining, ovarian tissue sections were deparaffinised, rehydrated and blocking and antigen retrieval performed as earlier. Mitochondrial encoded cytochrome c oxidase (MTCO1) (1D6E1A8) mouse monoclonal (1:1000; Abcam ab14705, Melbourne, Australia) antibody, lysosomeassociated membrane protein 1 (LAMP-1) rat monoclonal anti-mouse antibody (1D4B) (1:100; Developmental Studies Hybridoma Bank, lowa, USA), or phospho-Histone $(\gamma)$ H2AX (Ser139) rabbit monoclonal (1:100; Cell Signaling Technology \#9718) primary antibody was applied in 1\% BSA/ TNT overnight at $4{ }^{\circ} \mathrm{C}$. Tissues were incubated with Alexa568conjugated goat secondary antibodies to mouse, rat or rabbit $\operatorname{lgG}$ (1:400; Molecular Probes) in 1\% BSA/TNT for $1 \mathrm{~h}$ at room temperature. Sections were mounted with ProLong Diamond Anti-Fade Mountant containing DAPI (ThermoFisher). Slides were analysed by fluorescent confocal microscopy using a Nikon C1 Upright Confocal microscope. MTCO1-positive mitochondrial staining patterns were assessed by a blinded reviewer and categorically defined as either homogenous (based on a clear even distribution staining pattern throughout the ooplasm) or abnormal (based on the clear presence of vacuoles and uneven staining pattern throughout the ooplasm), and then expressed as a proportion (\%) of total follicles. Positive $\gamma \mathrm{H} 2 \mathrm{AX}$ follicles were defined by positive staining in 
the oocyte and/or $\geq 4$ granulosa cells when the ooplasm was visualised and expressed as a proportion (\%) of total follicles.

\section{Statistical analysis}

Data are presented as mean \pm S.E.M. and statistical analysis was performed using GraphPad Prism Software. Normally distributed data were analysed by Student's $t$-test to compare two groups. Data that were not normally distributed were compared using a Mann-Whitney test. The offspring sample size refers to the number of litters from which one female was analysed in each litter. Differences were considered significant when $P<0.05$.

\section{Results}

\section{Low-protein diet does not alter maternal ovarian follicle morphology or number}

Female mice (10 weeks) were randomised onto normal chow control diet or low-protein diet, and then mated 3 weeks later. Protein-restricted females were lighter than females fed control diet at mating (data not shown), although females were not weighed at plugging, as they were not time mated. The time to litter since pairing with a male stud was significantly increased in female mice fed low-protein diet vs mice fed control diet (control 21.7 days \pm 0.4 vs low-protein diet $30.8 \pm 3.9, P<0.05$ ) (Fig. 1A). However, there were no differences in the number of live pups born between groups (Fig. 1B). There was no difference in sex ratio of the offspring between diet groups (data not shown), although lowprotein diet offspring were lighter than control offspring at PN2 (control $1.52 \pm 0.06 \mathrm{~g}$ vs low-protein diet $1.26 \pm 0.03 \mathrm{~g}, P<0.05)$. Pups were not handled prior to this to ensure that the mother did not reject the litter. Mothers were maintained on the diets throughout pregnancy and lactation until weaning at PN21, at which time maternal ovaries were harvested. There were no overall morphological effects of low-protein diet on maternal ovaries compared to control diet (Fig. 1C and D). Quantification of healthy primordial, transitional, primary, secondary and antral ovarian follicles were based upon the follicle morphology shown in representative images (Fig. 1E, F, G, H and I). The numbers of each follicle class in the two diet groups was similar (Fig. 1J). Likewise, ovarian volume (Fig. 1K) and corpora lutea number (Fig. 1L), indicative of ovulation, were similar in the two groups. However, maternal weight at PN21 was significantly lower in mothers fed a low-protein diet than control mothers (Fig. 1M).

\section{Low-protein diet results in low ovarian reserve of primordial follicles in PN21 offspring}

There were no morphological differences in ovaries between the two groups at PN21 (Fig. 2A and B).
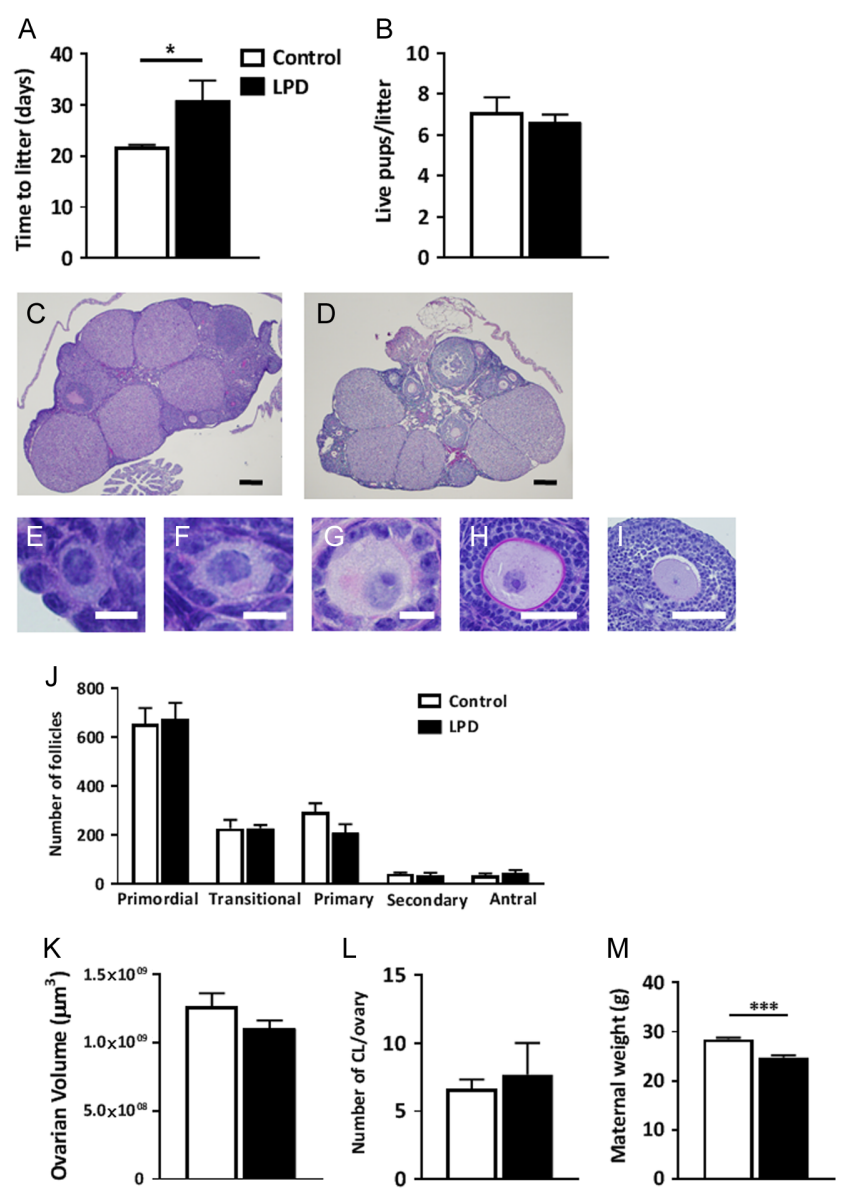

Figure 1 Analysis of maternal pregnancy and ovaries. (A) The time to litter since pairing with a male stud was recorded in days $(n=11-12$ / group). (B) The number of live pups per litter was recorded ( $n=11-12$ /group). Maternal ovaries ( 18 weeks; $n=5 /$ group) from mice fed (C) control diet or (D) low-protein diet were randomly selected and stained with PAS. Bars are $200 \mu \mathrm{m}$. Representative images of (E) primordial follicle oocyte; bar $=10 \mu \mathrm{m}$, (F) transitional follicle; bar $=10 \mu \mathrm{m},(\mathrm{G})$ primary follicle bar $=20 \mu \mathrm{m}$, (H) secondary follicle; bar $=50 \mu \mathrm{m}$, and (I) antral follicle; bar $=100 \mu \mathrm{m}$. (J) Total numbers of primordial, transitional, primary, secondary and antral follicles per ovary, $(\mathrm{K})$ ovarian volume $\left(\mu \mathrm{m}^{3}\right)$, (L) numbers of corpora lutea per ovary and $(M)$ maternal weight was recorded following weaning. Data are mean \pm S.E.M., $t$-test, ${ }^{*} P<0.05,{ }^{* * *} P<0.001$.

However, upon quantification of each follicle class, the number of primordial follicle oocytes in offspring from mothers fed the low-protein diet was 37\% lower than that in control offspring (control $3283 \pm 216$ vs low-protein diet $2063 \pm 383$ primordial follicles/ovary, $P<0.05)$ (Fig. 2C). There were no differences in the number of all other follicle classes between groups (Fig. 2C) and ovarian volume was similar in the two groups (Fig. 2D). Low-protein diet offspring body weight at PN21 was $18 \%$ less than that of control offspring (control $9.41 \mathrm{~g} \pm 0.20$ vs low-protein diet $7.70 \mathrm{~g} \pm 0.38$, $P<0.01$ ) (Fig. 2E). 


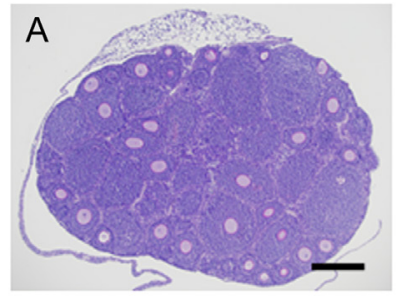

C

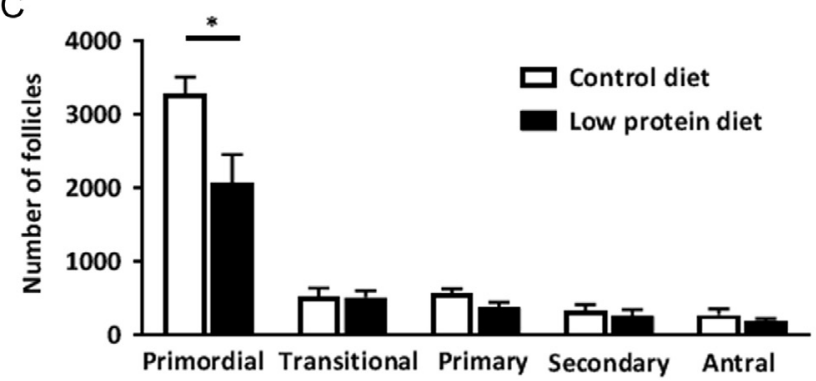

$\mathrm{D}$

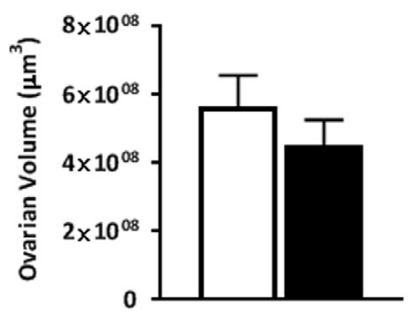

E

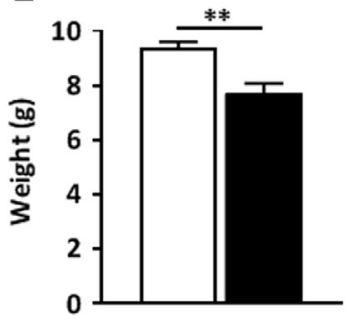

Figure 2 Analysis of offspring ovaries at PN21. PAS stained PN21 ovaries from offspring of mothers fed (A) control diet or (B) lowprotein diet. Bars are $200 \mu \mathrm{m}$. (C) Total numbers of primordial, transitional, primary, secondary and antral follicles, (D) ovarian volume $\left(\mu \mathrm{m}^{3}\right)$ and (E) offspring weight at PN21. Data are mean \pm S.E.M., $t$-test, $n=5$ mice/group; $* P<0.05$.

\section{Low-protein diet reduces the reserve of primordial follicles in adult offspring}

Ovaries from offspring of mothers fed control or low-protein diet were harvested at 24 weeks of age. Ovaries from the low-protein diet group appeared morphologically similar to controls (Fig. 3A and B). However, there were $51 \%$ fewer primordial follicle oocytes in offspring from mothers fed low-protein diet vs control (control $777 \pm 101$ vs. low-protein diet $383 \pm 95$ follicles/ovary, $P<0.05$ ) (Fig. 3C). There were no differences in the numbers of the other follicle classes (Fig. 3C). Ovarian volume was similar in the two groups (Fig. 2D). The number of corpora lutea was significantly lower in the low-protein diet group than in the control group (control $7.2 \pm 0.66$ vs. low-protein diet $4.0 \pm 0.63, P<0.01$ ) (Fig. 3E). Offspring weight at 24 weeks was similar in the two groups (Fig. 4F).
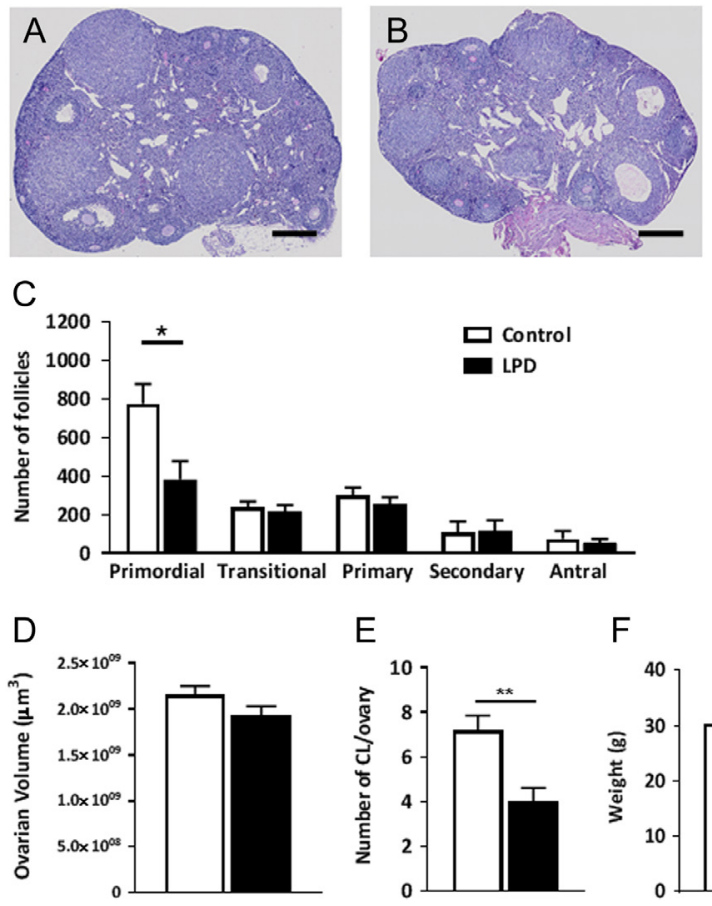

$\mathrm{E}$

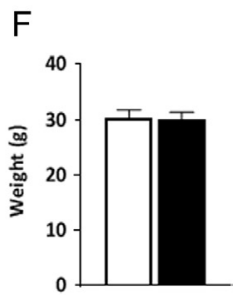

Figure 3 Analysis of adult offspring ovaries at 24 weeks. PAS-stained ovaries from adult offspring of mothers fed (A) control diet or (B) low-protein diet. Bars are $200 \mu \mathrm{m}$. (C) Total numbers of primordial, transitional, primary, secondary and antral follicles (D), ovarian volume $\left(\mu \mathrm{m}^{3}\right)$ and $(\mathrm{F})$ corpora lutea were quantified and (E) offspring weight recorded at 24 weeks. Data are mean \pm S.E.M., $t$-test, $n=5$ mice/group; ${ }^{*} P<0.05,{ }^{* *} P<0.01$.

\section{Maternal low-protein diet increases offspring granulosa cell apoptosis in mice, but does not alter proliferation}

Apoptosis was assessed by TUNEL staining (Fig. 4A, B, C, $\mathrm{D}, \mathrm{E}, \mathrm{F}, \mathrm{G}$ and $\mathrm{H}$ ) and cleaved caspase- 3 immunostaining (Fig. 4I, J, K, L, M, N, O and P) in PN21 and 24-weekold offspring ovaries from mothers fed control or lowprotein diet. TUNEL and cleaved caspase-3 staining were consistently absent in primordial, transitional and primary follicles from both groups, at both ages. TUNEL and cleaved caspase-3 were primarily localised to the granulosa cells of secondary and antral follicles. In all experiments, a negative control was employed in which the primary antibody or enzyme was omitted and no positive staining was detected in these sections, highlighting the staining specificity. Representative negative controls are shown (inset). The proportions of TUNEL-positive (Fig. 4Q) and cleaved caspase-3-positive follicles (Fig. 4R) were significantly increased in ovaries from offspring exposed to maternal low-protein diet vs control at PN21 and at 24 weeks $(P<0.05, P<0.01)$. Proliferation was monitored using $\mathrm{pH} 3$ staining. Positive immunostaining for $\mathrm{pH} 3$ was undetectable in primordial 



Figure 4 Analysis of follicle atresia. Representative photomicrographs of TUNEL stained ovaries from PN21 offspring (A, B, C and D) or adult offspring (E, F, G and H) of mothers fed control or low-protein diet. Representative photomicrographs of cleaved caspase-3 immunostained ovaries from PN21 offspring ( $\mathrm{I}, \mathrm{J}, \mathrm{K}$ and $\mathrm{L})$ or adult offspring $(\mathrm{M}, \mathrm{N}, \mathrm{O}$ and $\mathrm{P})$ of mothers fed control or low-protein diet. Insets are negative controls. Bars are $500 \mu \mathrm{m}(\mathrm{A}, \mathrm{B}, \mathrm{E}, \mathrm{F}, \mathrm{I}, \mathrm{J}, \mathrm{M}, \mathrm{N})$, or $200 \mu \mathrm{m}(\mathrm{C}, \mathrm{D}, \mathrm{G}, \mathrm{H}, \mathrm{K}, \mathrm{L}, \mathrm{O}, \mathrm{P})$. The number of (Q) TUNEL and (R) cleaved caspase-3 positive stained follicles were quantified in 3-5 sections per ovary and expressed as a percentage of total follicles. Positive follicles were defined by positive oocyte staining and/or $\geq 4$ positive granulosa cells per follicle, where the ooplasm was visualised. Data are mean \pm S.E.M., $t$-test, $n=5$ mice/group; ${ }^{*} P<0.05,{ }^{* *} P<0.01$. 

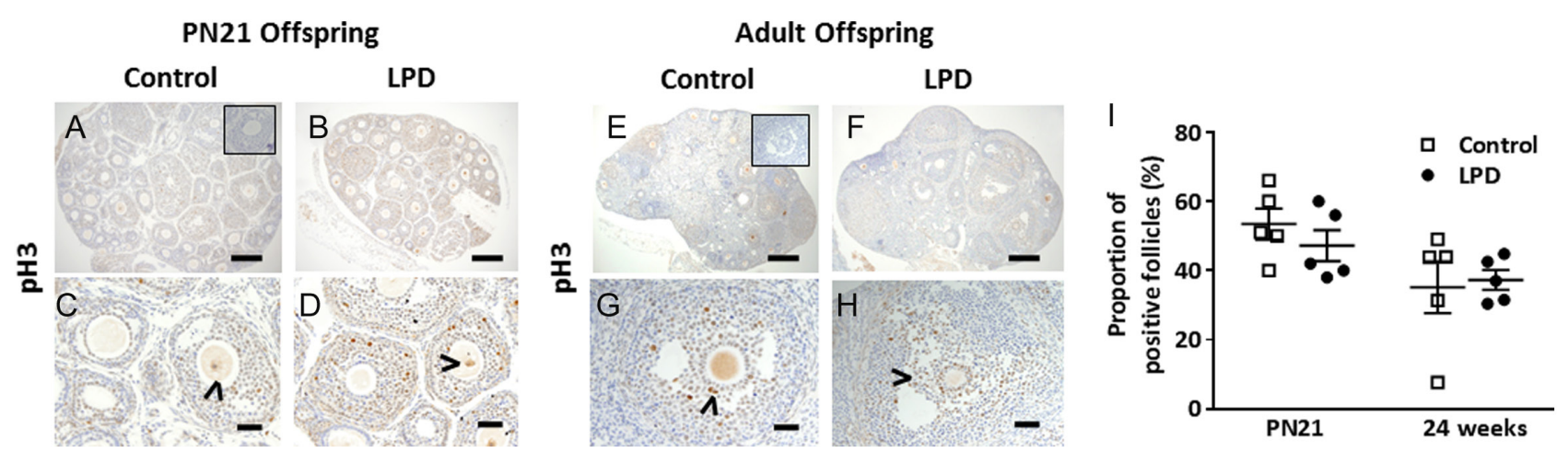

Figure 5 Analysis of follicle proliferation. Representative photomicrographs of phosphorylated histone $\mathrm{H} 3$ ( $\mathrm{pH} 3$ ) immunostained ovaries from PN21 offspring (A, B, C and D) or adult offspring (E, F, G and H) of mothers fed control diet or low-protein diet. Insets are negative controls. Bars are $500 \mu \mathrm{m}(\mathrm{A}, \mathrm{B}, \mathrm{E}, \mathrm{F})$, or $200 \mu \mathrm{m}(\mathrm{C}, \mathrm{D}, \mathrm{G}, \mathrm{H})$. (I) The number of $\mathrm{pH} 3$ positive stained follicles was quantified in $3-5$ sections per ovary and expressed as a proportion of total follicles (\%). Positive follicles were defined by positive oocyte staining and/or $\geq 4$ positive granulosa cells per follicle, where the ooplasm was visualised. Data are mean \pm S.E.M., $t$-test, $n=5$ mice/group.

and transitional follicles and rarely present in primary follicles, likely due to the low mitotic index of these cells. Positive $\mathrm{pH} 3$ immunostaining was frequently evident in the granulosa cells of secondary and antral follicles in both offspring groups, at both ages (Fig. 5A, B, C, D, E, $F, G$ and $H$ ). Though there were no differences in the proportions of $\mathrm{pH} 3$-positive follicles between treatment groups at either age (Fig. 5I).

\section{Maternal low-protein diet is associated with increased vacuoles in oocytes}

To assess whether maternal low-protein diet affected mitochondrial distribution within offspring follicles, MTCO1 was used as a marker to highlight mitochondrial cytochrome c oxidase, required for mitochondrial oxidative phosphorylation in ovarian tissue sections. Positive MTCO1 immunostaining localised to the ooplasm of growing secondary and antral follicles in ovarian tissues from both groups, at both ages. However, altered mitochondrial distribution patterns within the ooplasm were evident. To analyse this, we categorically classified the staining patterns observed as either homogenous normal distribution (arrow heads) (Fig. 6A and C) or abnormal distribution (arrows) (Fig. 6B and D). At PN21, we found a significantly lower percentage of follicles with homogenous mitochondrial distribution in offspring from mothers fed low-protein diet (control $80.9 \% \pm 5.8$ vs. low-protein diet $36.0 \% \pm 4.4$ follicles/ ovary, $P<0.0001)$, and a subsequent higher proportion of follicles with abnormal mitochondrial distribution compared to control (control $19.1 \% \pm 5.5$ vs low-protein diet $64.0 \% \pm 4.1$ follicles/ovary, $P<0.05$ ) (Fig. 6E). However, by 24 weeks of age, there were no differences in offspring oocyte mitochondrial distribution between groups (Fig. 6E). Morphologically, the abnormal patterns of mitochondrial distribution appeared to be potentially attributed to the presence of a large number of vacuoles.
LAMP-1 is one of the most abundant proteins that forms lysosomal membranes. Therefore, LAMP-1 immunofluorescence was performed to determine if these alterations were due to lysosome formation. However, the patterns of lysosome staining (Fig. 6F, G, $\mathrm{H}$ and $\mathrm{I}$, white arrow heads) did not correspond with the presence of apparent vacuoles (Fig. 6G and I, yellow outline). In all experiments, a negative control was employed in which the primary antibody or enzyme was omitted and no positive staining was detected in these sections, highlighting the specificity of the staining. Representative negative controls are shown (inset).

\section{Maternal low-protein diet increases adult offspring follicle DNA damage}

To assess whether maternal low-protein diet contributed to DNA damage within offspring follicles, $\gamma \mathrm{H} 2 \mathrm{AX}$ immunostaining was performed. Positive $\gamma \mathrm{H} 2 \mathrm{AX}$ localisation indicates the presence of DNA doublestrand breaks and the commencement of DNA damage repair. Little-to-no $\gamma \mathrm{H} 2 \mathrm{AX}$-positive immunostaining was detected in control diet ovaries at PN21 (Fig. 7A) or at 24 weeks of age (Fig. 7C). When positive staining was detected in control sections, $\gamma \mathrm{H} 2 \mathrm{AX}$ foci were only detected in growing secondary or antral follicles and primarily localised to the granulosa cells, with occasional staining detected in oocyte nuclei (Fig. 7A and $\mathrm{C}$ ). In contrast, positive $\gamma \mathrm{H} 2 \mathrm{AX}$ foci localised to primordial, primary, secondary and antral follicles in ovaries from offspring of mothers fed low-protein diet animals at both ages, though the proportion of follicles with positive staining was quite variable between animals (Fig. 7B and D). Positive $\gamma \mathrm{H} 2 \mathrm{AX}$ foci detected in protein-restricted offspring follicles localised to both oocyte nuclei and to granulosa cells. At PN21, there was no significant difference in the proportion of $\gamma \mathrm{H} 2 \mathrm{AX}$ positive follicles between groups (Fig. 7E), however, by 
PN21

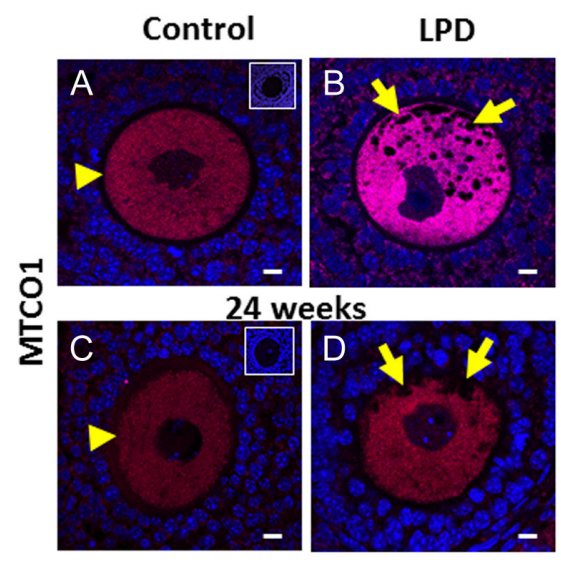

PN21

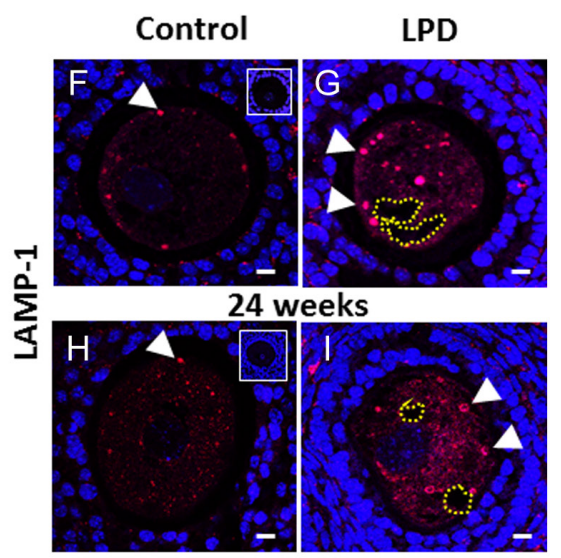

Figure 6 Analysis of follicle mitochondrial and lysosome distribution. Representative photomicrographs of MTCO1 immunostained ovaries from PN21 (A and B) and adult offspring (C and D) of mothers fed control diet or low-protein diet. Follicles with positive staining and ooplasm present were categorised based on homogenous (arrow head), or abnormal (arrow) mitochondrial staining. (E) The number of follicles with homogenous or abnormal staining was expressed as the proportion of total follicles (\%), analysed in 3-5 sections per ovary. 465 oocytes were analysed in 20 mice ( $n=5 /$ group). Data are mean \pm S.E.M., $t$-test, $* * * P<0.001$.

Representative photomicrographs of LAMP-1 immunofluorescence from PN21 (F and G) or adult offspring ( $\mathrm{H}$ and $\mathrm{I}$ ) of mothers fed control diet, or low-protein diet. Lysosomes (positive staining, white arrow heads) did not correspond with apparent vacuoles (yellow outline) in low-protein diet offspring oocytes. Insets are negative controls. Bars are $10 \mu \mathrm{m}$.

24 weeks, the proportion of $\gamma \mathrm{H} 2 \mathrm{AX}$-positive follicles was significantly increased in the low-protein diet group compared to control (control $6.7 \% \pm 3.1 \mathrm{vs}$ low-protein diet $21.8 \% \pm 5.6$ follicles/ovary, $P<0.05$ ) (Fig. 7E).

\section{Discussion}

It is well established that the early life environment plays a crucial role in determining the health outcomes of offspring in later life (Gluckman et al. 2008), a phenomenon which extends to the development of the female reproductive system (Aiken et al. 2016). Evidence from animal models and women show that maternal protein restriction during pregnancy and lactation impairs the development of the offspring ovarian reserve. This is the first study to examine the combined effect of preconception, in utero and prepubertal protein restriction on ovarian follicle endowment in vivo. We report here that the ovarian reserve of primordial follicles, an important determinant of female fertile lifespan, is significantly reduced in offspring from mothers fed lowprotein diet prior to and during pregnancy and lactation, compared to offspring from mothers fed a control diet.

Protein restriction significantly decreased maternal body weight, but had no effect on the numbers of follicles or corpora lutea, suggesting normal ovulation, although this was not directly assessed. A reduction in oocyte quality is commonly indicated by a reduction in litter size in breeding studies, which requires more than one round of mating to determine in mice (Kerr et al. 2012). However, we found no differences in litter sizes between groups. Interestingly though, the time to litter was significantly increased in female mice fed lowprotein diet, vs control. This suggests a reduction in maternal oocyte quality, or alternatively could reflect the effects of stress, or other factors which impacted on mating behaviour. Previously, calorie restriction was reported to delayed estrous cycling in mice (Bronson \& Marsteller 1985, Schubert et al. 2008). Therefore, it is possible that protein restriction may have contributed to delayed time to litter, although we did not examine the impacts of maternal protein restriction on estrous cycling in the mothers or the offspring. Additionally, the low-protein chow used in the present study contains double the amount of sucrose as the normal chow diet. Sucrose is composed of both glucose and fructose. Notably, in women, high sugar intake prior to oocyte retrieval and IVF has been correlated with reduced oocyte retrieval, impaired oocyte maturation, reduced fertilisation rate and abnormal embryo morphology (Machtinger et al. 2017).

To address the primary aim of this study, offspring ovarian follicles were enumerated. Our data show that maternal low-protein diet led to a striking 37\% and $51 \%$ fewer primordial follicles in juvenile PN21 and adult 24-week-old offspring, respectively. This finding indicates that exposure to an early life insult, such as protein restriction has a profound impact on the ovarian reserve, which may become more pronounced with advancing age in offspring. It is difficult however to compare our findings with previous rat models, given the experimental differences in the timing of maternal protein 

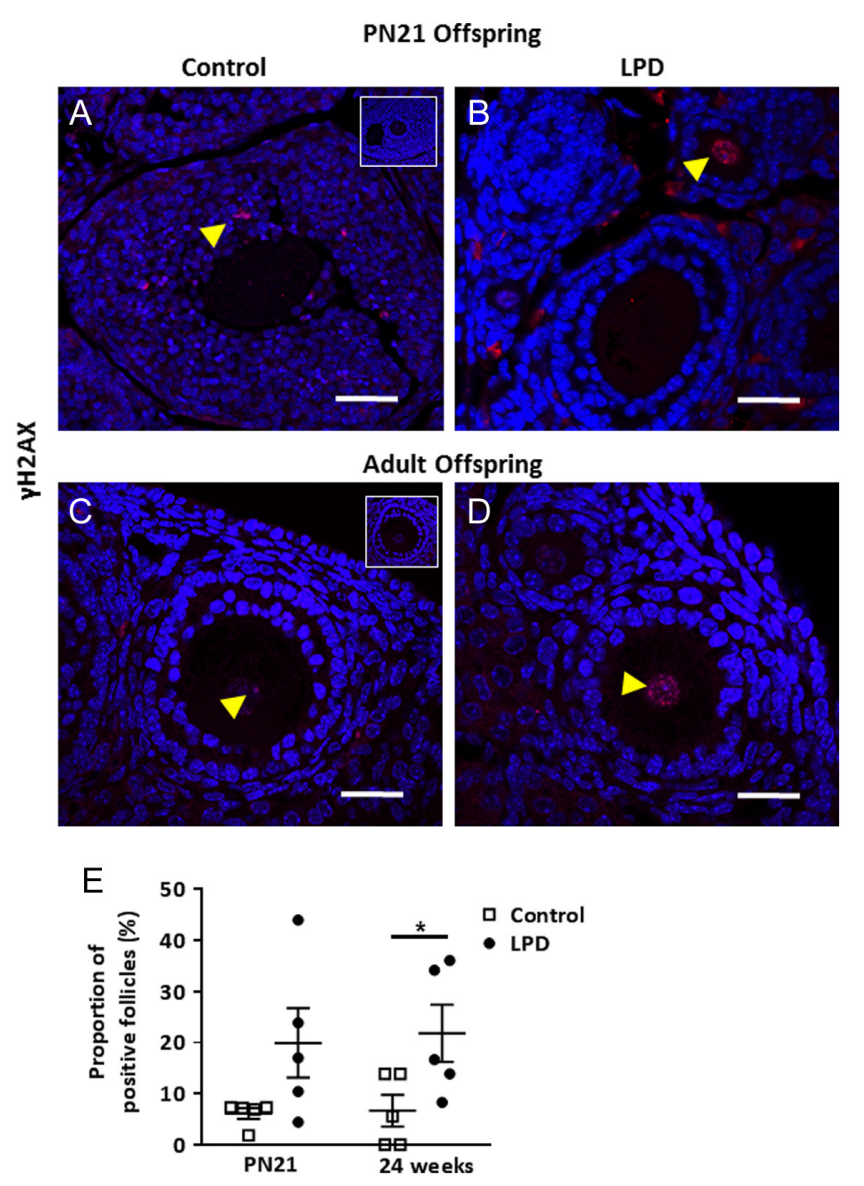

Figure 7 Analysis of follicle DNA damage. Representative photomicrographs of $\gamma \mathrm{H} 2 \mathrm{AX}$ immunostained ovaries from PN21 (A and $\mathrm{B}$ ) or adult offspring ( $\mathrm{C}$ and $\mathrm{D})$ of mothers fed control diet or lowprotein diet. Insets are negative controls. Bars are $200 \mu \mathrm{m}$. (E) The number of $\gamma \mathrm{H} 2 \mathrm{AX}$-positive stained follicles was quantified in 3-5 sections per ovary and expressed as a proportion of total follicles (\%). Positive follicles were defined by positive oocyte staining and/or $\geq 4$ positive granulosa cells per follicle, where the nucleus was visualised. 455 oocytes were analyses in 20 mice ( $n=5 /$ group). Data are mean \pm S.E.M., Mann-Whitney test (PN21), $t$-test (24 week), $* P<0.05$.

restriction and technical differences in the methods used to analyse follicles. Unlike previous reports of maternal protein restriction on offspring ovarian reserve, which measured follicle density (Bernal et al. 2010, da Silva Faria et al. 2010a,b, Guzman et al. 2014), we have estimated total follicle numbers as previously detailed (Tilly 2003). We recently reported that follicle density values do not correspond with total follicle estimates calculated in the same mouse ovary (Winship et al. 2018). The quantification of follicle density is a less robust measure than total follicle estimates, as measures of density do not account for the unequal distribution of follicles throughout the ovary or fluctuations in ovarian volume.

The lower ovarian reserve identified in the present study could be due to any or all of the following: fewer granulosa cells or oocytes being generated during in utero ovarian development, or increased apoptosis during oogenesis, or decreased follicle formation after establishment of the primordial follicle reserve postnatally. Our study is limited by the absence of lategestational or early postnatal ovarian samples from protein-restricted mothers, which may help to enable us to pinpoint the window of follicle loss. Therefore, further mechanistic studies should be performed to narrow the window of follicle loss in protein-restricted offspring. We sought to gain some insight into the molecular changes underlying follicle loss in protein-restricted offspring using immunohistochemical and histological analyses. Although our results show fewer primordial follicles in offspring exposed to maternal protein restriction, TUNEL and cleaved caspase- 3 analysis failed to detect evidence of increased apoptosis within primordial follicles in offspring at either age. The mechanism of offspring primordial follicle depletion therefore remains unknown; however, it cannot be ruled out that protein restriction causes direct primordial follicle apoptosis, as previous studies have also reported the inability of current markers to identify apoptotic primordial follicles, even during periods of dramatic follicle loss (Tingen et al. 2009, Liew et al. 2014, 2017).

The overall proportion of follicles with apoptotic granulosa cells was however significantly increased in low-protein diet offspring compared to controls at both ages. Cleaved caspase-3 and TUNEL-positive staining were predominantly evident in growing secondary and antral follicles and suggest increased follicle atresia. Surprisingly though, there were no differences in the numbers of secondary or antral follicles between the two groups. Thus, it is possible that the increase in granulosa cell apoptosis was insufficient to induce follicle death. Alternatively, these contrasting findings may be attributed to an increase in primordial follicle activation to bolster growing follicle numbers in the lowprotein group, which is consistent with a depletion of the ovarian reserve of primordial follicles that we have observed. Previous studies investigating preantral and antral follicles found that maternal protein restriction during either pregnancy or lactation alone impaired offspring follicle counts compared to controls in rats at PN21 (Guzman et al. 2014). Interestingly though, in support of our findings, protein restriction for an extended time, during both pregnancy and lactation combined, had no effect on preantral or antral follicle numbers (Guzman et al. 2014). Since primordial follicles were not assessed in this study though, it is difficult to speculate on the mechanism of this difference.

A previous report has shown mitochondrial dysfunction, characterised by reduced membrane potential, and increased calcium and reactive oxygen species (ROS) levels, in mouse embryos from proteinrestricted mothers (Mitchell et al. 2009). Although we did not analyse mitochondrial function, in our study, 
we investigated mitochondrial distribution using the marker, MTCO1, which is the terminal component of the mitochondrial respiratory chain. Mitochondrial distribution within developing oocytes is important for ATP production and oocyte competence and homogenous distribution throughout the ooplasm has been reported in healthy oocytes (Jin \& Kim 2017). Mitochondrial distribution appeared homogenous throughout the ooplasm in the majority of growing follicles in control offspring. However, MTCO1 staining patterns were significantly altered in growing follicles, including secondary and antral follicles in low-protein diet offspring. In these oocytes, MTOC1 staining appeared to be excluded from large zones of the ooplasm.

The meaning of this abnormal staining pattern in terms of mitochondrial function is unclear, but may be attributed to the presence of vacuoles. In intermediate stages of cell death, numerous clear vacuoles of different size are commonly observed in oocyte cytoplasm (Ortiz et al. 2006). Whilst we did not co-localise markers of mitochondria, or vacuoles with markers of apoptosis, we did examine the localisation of LAMP-1 to determine whether the presence of vacuoles may be attributed to increased lysosomes, intermediate autophagosomes or autolysosomes formation (Kirkegaard et al. 2004). The presence of abundant lysosomes in the cytoplasm was demonstrated predominantly in growing follicles from low-protein diet offspring. However, the staining pattern did not correspond with the presence of vacuoles observed in MTCO1-stained sections. It is therefore possible that this morphology is instead due to the presence of lipid accumulation in the ooplasm. Indeed, a characteristic of apoptosis is the rapid accumulation of cytoplasmic lipid droplets associated with impaired mitochondrial function and an increase in ROS (Boren \& Brindle 2012).

We subsequently investigated the impact of maternal low-protein diet on offspring ovarian follicle DNA damage, by performing immunofluorescence using the marker $\gamma \mathrm{H} 2 \mathrm{AX}$, which forms quantifiable staining at the sites of DNA double-strand breaks. Maternal protein restriction led to increased DNA damage in growing follicles in offspring ovarian tissue at 24 weeks; however, there were no differences at PN21, likely due to high variability. Maternal protein restriction has previously been shown to cause DNA damage in rat offspring cardiac tissue (Blackmore et al. 2012), though this is the first report in the ovary. The mechanism of follicular DNA damage is unclear. It is possible that lowprotein diet offspring ovaries are subject to increased levels of ROS accumulation, although this remains to be investigated.

Previously, delayed sexual maturation and premature ageing of reproductive function were reported in adult offspring rats from mothers fed a protein-restricted diet during lactation or both pregnancy and lactation (Guzman et al. 2006). However, surrogate measures of reproductive lifespan, including estrous cycle length were used in that study and the ovarian reserve was not examined. Although breeding studies were carried out, these were not robust and occurred within one estrous cycle at two time points; female rats were paired with males at $\sim 160$ and $\sim 360$ days. A reduction in oocyte quality is reflected in a reduction in litter size in breeding studies. Whilst not significant, Guzman et al. reported an overall mean reduction of one pup per litter in 150-day-old rat offspring from mothers fed lowprotein diet vs control diet (2006). Furthermore, when the breeding study was repeated when offspring reached 1 year of age, there was a significant reduction in litter size, suggesting premature loss of the ovarian reserve and a possible reduction in oocyte quality. Together, these data suggest that maternal protein restriction may influence female offspring fertility, although a clear limitation of our study and others is the lack of robust fertility trials. In order to definitively determine whether the diminished ovarian reserve reported in the present study translates to a reduced fertile lifespan, breeding studies involving multiple mating rounds need to be performed as previously described (Nguyen et al. 2018).

The maternal low-protein diet model during gestation and lactation is one of the most extensively characterised animal models used to study of the effects of nutrient deficiency on offspring phenotype (reviewed Kereliuk et al. 2017). Mounting evidence from women (Yarde et al. 2013) and animal models support our findings, which suggest that the preconception diet may also play a key role in determining the health of offspring. Preconception protein restriction for 2 weeks prior to mating impaired placental function and offspring growth in mice (Gonzalez et al. 2016). Furthermore, maternal protein restriction fed exclusively for one ovulatory cycle prior to mating (Watkins et al. 2008) or only during preimplantation embryo development (Watkins et al. 2011) led to adverse cardiovascular development in offspring. Although we did not investigate the impact of maternal protein restriction during the preconception period alone in this study, the dramatic proportions of primordial follicle depletion that we observed in lowprotein diet offspring suggest that nutrient availability during maternal oocyte maturation may contribute to the programming offspring ovarian reserve and this window of development requires furthers study.

In Australia, current National Health and Medical Research Council dietary guidelines are available for women during pregnancy (NHMRC 2013). However, emerging evidence, including our present findings, highlight the potential benefits for creating specific dietary guidelines and also improving clinical and public awareness of the importance of preconception diet for expectant mothers to develop healthy offspring. 


\section{Declaration of interest}

The authors declare that there is no conflict of interest that could be perceived as prejudicing the impartiality of the research reported.

\section{Funding}

A W was supported by a NHMRC Early Career Fellowship (1120300).

\section{Acknowledgements}

The authors would like to acknowledge the technical support of the Monash Animal Research Laboratories, Monash Histology Platform and Monash Micro Imaging Facility. This work was made possible through Victorian State Government Operational Infrastructure Support and Australian Government NHMRC IRIISS.

\section{References}

Aiken CE, Tarry-Adkins JL \& Ozanne SE 2016 Transgenerational effects of maternal diet on metabolic and reproductive ageing. Mammalian Genome 27 430-439. (https://doi.org/10.1007/s00335-016-9631-1)

Bernal AB, Vickers MH, Hampton MB, Poynton RA \& Sloboda DM 2010 Maternal undernutrition significantly impacts ovarian follicle number and increases ovarian oxidative stress in adult rat offspring. PLOS ONE 5 e15558. (https://doi.org/10.1371/journal.pone.0015558)

Blackmore HL, Piekarz AV, Fernandez-Twinn DS, Mercer JR, Figg N, Bennett M \& Ozanne SE 2012 Poor maternal nutrition programmes a pro-atherosclerotic phenotype in ApoE-/- mice. Clinical Science 123 251-257. (https://doi.org/10.1042/CS20110487)

Boren J \& Brindle KM 2012 Apoptosis-induced mitochondrial dysfunction causes cytoplasmic lipid droplet formation. Cell Death and Differentiation 19 1561-1570. (https://doi.org/10.1038/cdd.2012.34)

Bronson FH \& Marsteller FA 1985 Effect of short-term food deprivation on reproduction in female mice. Biology of Reproduction 33 660-667. (https://doi.org/10.1095/biolreprod33.3.660)

da Silva Faria T, de Bittencourt Brasil F, Sampaio FJ \& da Fonte Ramos C $2010 \mathrm{a}$ Effects of maternal undernutrition during lactation on estrogen and androgen receptor expressions in rat ovary at puberty. Nutrition $\mathbf{2 6}$ 993-999. (https://doi.org/10.1016/j.nut.2009.09.027)

da Silva Faria T, de Bittencourt Brasil F, Sampaio FJ \& da Fonte Ramos C $2010 \mathrm{~b}$ Maternal malnutrition during lactation affects folliculogenesis, gonadotropins, and leptin receptors in adult rats. Nutrition $\mathbf{2 6}$ 1000-1007. (https://doi.org/10.1016/j.nut.2009.09.026)

Findlay JK, Hutt KJ, Hickey M \& Anderson RA 2015 How is the number of primordial follicles in the ovarian reserve established? Biology of Reproduction 93 111. (https://doi.org/10.1095/biolreprod.115.133652)

Flaws JA, Abbud R, Mann RJ, Nilson JH \& Hirshfield AN 1997 Chronically elevated luteinizing hormone depletes primordial follicles in the mouse ovary. Biology of Reproduction 57 1233-1237. (https://doi.org/10.1095/ biolreprod57.5.1233)

Gluckman PD, Hanson MA, Cooper C \& Thornburg KL 2008 Effect of in utero and early-life conditions on adult health and disease. New England Journal of Medicine 359 61-73. (https://doi.org/10.1056/ NEJMra0708473)

Gonzalez PN, Gasperowicz M, Barbeito-Andres J, Klenin N, Cross JC \& Hallgrimsson B 2016 Chronic protein restriction in mice impacts placental function and maternal body weight before fetal growth. PLoS ONE 11 e0152227. (https://doi.org/10.1371/journal.pone.0152227)

Guzman C, Cabrera R, Cardenas M, Larrea F, Nathanielsz PW \& Zambrano E 2006 Protein restriction during fetal and neonatal development in the rat alters reproductive function and accelerates reproductive ageing in female progeny. Journal of Physiology $\mathbf{5 7 2}$ 97-108. (https://doi.org/10.1113/jphysiol.2005.103903)
Guzman C, Garcia-Becerra R, Aguilar-Medina MA, Mendez I, MerchantLarios H \& Zambrano E 2014 Maternal protein restriction during pregnancy and/or lactation negatively affects follicular ovarian development and steroidogenesis in the prepubertal rat offspring. Archives of Medical Research 45 294-300. (https://doi.org/10.1016/j. arcmed.2014.05.005)

Hutt KJ, McLaughlin EA \& Holland MK 2006 KIT/KIT ligand in mammalian oogenesis and folliculogenesis: roles in rabbit and murine ovarian follicle activation and oocyte growth. Biology of Reproduction 75 421-433. (https://doi.org/10.1095/biolreprod.106.051516)

Jin ZL \& Kim NH 2017 RAD51 maintains chromosome integrity and mitochondrial distribution during porcine oocyte maturation in vitro. Journal of Reproduction and Development 63 489-496. (https://doi. org/10.1262/jrd.2017-078)

Karbalay-Doust S \& Noorafshan A 2012 Stereological estimation of ovarian oocyte volume, surface area and number: application on mice treated with nandrolone decanoate. Folia Histochemica et Cytobiologica 50 275-279. (https://doi.org/10.5603/FHC.2012.0037)

Kereliuk SM, Brawerman GM \& Dolinsky VW 2017 Maternal macronutrient consumption and the developmental origins of metabolic disease in the offspring. International Journal of Molecular Sciences 18 E1451. (https:// doi.org/10.3390/ijms18071451)

Kerr JB, Hutt KJ, Cook M, Speed TP, Strasser A, Findlay JK \& Scott CL 2012 Cisplatin-induced primordial follicle oocyte killing and loss of fertility are not prevented by imatinib. Nature Medicine 18 1170-1172. (https:// doi.org/10.1038/nm.2889)

Kirkegaard K, Taylor MP \& Jackson WT 2004 Cellular autophagy: surrender, avoidance and subversion by microorganisms. Nature Reviews Microbiology 2 301-314. (https://doi.org/10.1038/ nrmicro865)

Liew SH, Vaithiyanathan K, Cook M, Bouillet P, Scott CL, Kerr JB, Strasser A, Findlay JK \& Hutt KJ 2014 Loss of the proapoptotic BH3only protein $\mathrm{BCL}-2$ modifying factor prolongs the fertile life span in female mice. Biology of Reproduction 90 77. (https://doi.org/10.1095/ biolreprod.113.116947)

Liew SH, Nguyen QN, Strasser A, Findlay JK \& Hutt KJ 2017 The ovarian reserve is depleted during puberty in a hormonally driven process dependent on the pro-apoptotic protein BMF. Cell Death and Differentiation 8 e2971. (https://doi.org/10.1038/cddis.2017.361)

Machtinger R, Gaskins AJ, Mansur A, Adir M, Racowsky C, Baccarelli AA, Hauser R \& Chavarro JE 2017 Association between preconception maternal beverage intake and in vitro fertilization outcomes. Fertility and Sterility 108 1026-1033. (https://doi.org/10.1016/j. fertnstert.2017.09.007)

McGee EA \& Hsueh AJ 2000 Initial and cyclic recruitment of ovarian follicles. Endocrine Reviews 21 200-214. (https://doi.org/10.1210/ edrv.21.2.0394)

Mitchell M, Schulz SL, Armstrong DT \& Lane M 2009 Metabolic and mitochondrial dysfunction in early mouse embryos following maternal dietary protein intervention. Biology of Reproduction 80 622-630. (https://doi.org/10.1095/biolreprod.108.072595)

Nguyen QN, Zerafa N, Liew SH, Morgan FH, Strasser A, Scott CL, Findlay JK, Hickey M \& Hutt KJ 2018 Loss of PUMA protects the ovarian reserve during DNA-damaging chemotherapy and preserves fertility. Cell Death and Differentiation 9 618. (https://doi.org/10.1038/s41419-0180633-7)

National Health and Medical Research Council 2013 Australian dietary guideliness. Canberra: National Health and Medical Research Council

Ortiz R, Echeverria OM, Salgado R, Escobar ML \& Vazquez-Nin GH 2006 Fine structural and cytochemical analysis of the processes of cell death of oocytes in atretic follicles in new born and prepubertal rats. Apoptosis 11 25-37. (https://doi.org/10.1007/s10495-005-3347-0)

Schubert KA, Vaanholt LM, Stavasius F, Demas GE, Daan S \& Visser GH 2008 Female mice respond differently to costly foraging versus food restriction. Journal of Experimental Biology 211 2214-2223. (https://doi. org/10.1242/jeb.017525)

Tilly JL 2003 Ovarian follicle counts - not as simple as 1, 2, 3. Reproductive Biology and Endocrinology 1 11. (https://doi.org/10.1186/1477-7827-1-11)

Tingen CM, Bristol-Gould SK, Kiesewetter SE, Wellington JT, Shea L \& Woodruff TK 2009 Prepubertal primordial follicle loss in mice is not due to classical apoptotic pathways. Biology of Reproduction 81 16-25. (https://doi.org/10.1095/biolreprod.108.074898) 
Wallace WH \& Kelsey TW 2010 Human ovarian reserve from conception to the menopause. PLOS ONE 5 e8772. (https://doi.org/10.1371/journal. pone.0008772)

Watkins AJ, Wilkins A, Cunningham C, Perry VH, Seet MJ, Osmond C Eckert JJ, Torrens C, Cagampang FR, Cleal J et al. 2008 Low protein diet fed exclusively during mouse oocyte maturation leads to behavioural and cardiovascular abnormalities in offspring. Journal of Physiology 586 2231-2244. (https://doi.org/10.1113/jphysiol.2007.149229)

Watkins AJ, Lucas ES, Wilkins A, Cagampang FR \& Fleming TP 2011 Maternal periconceptional and gestational low protein diet affects mouse offspring growth, cardiovascular and adipose phenotype at 1 year of age. PLOS ONE 6 e28745. (https://doi.org/10.1371/journal.pone.0028745)

Winship AL, Bakai M, Sarma U, Liew SH \& Hutt KJ 2018 Dacarbazine depletes the ovarian reserve in mice and depletion is enhanced with age. Scientific Reports 8 6516. (https://doi.org/10.1038/s41598-018-24960-5)
Yarde F, Broekmans FJ, van der Pal-de Bruin KM, Schonbeck Y, te Velde ER, Stein AD \& Lumey LH 2013 Prenatal famine, birthweight, reproductive performance and age at menopause: the Dutch hunger winter families study. Human Reproduction 28 3328-3336. (https://doi.org/10.1093/ humrep/det331)

Received 18 May 2018

First decision 13 June 2018

Revised manuscript received 26 June 2018

Accepted 6 July 2018 